\title{
Dehydrated Human Amnion/Chorion Membrane Allograft Promotes Cardiac Repair Following Myocardial Infarction
}

\author{
Jeremy J Lim ${ }^{1}$, James Fonger ${ }^{2}$ and ${ }^{*}$ Thomas J Koob $^{1}$ \\ ${ }^{1}$ MiMedx Group Inc., USA \\ ${ }^{2}$ Innovation Perspectives, USA
}

Submission: December 22, 2016; Published: January 10, 2017

*Corresponding author: Thomas J Koob, PhD, 1775 West Oak Common Court NE, Marietta, GA 30062, USA, Tel: (770) 651-9100;

Fax: (770) 590-3562; Email: tkoob@mimedx.com

\begin{abstract}
There is a rising need for novel therapies for the treatment of acute myocardial infarction (MI). Dehydrated human amnion/chorion membrane (dHACM) contains a unique array of regenerative cytokines and growth factors, involved in the regulation of tissue healing and modulation of inflammation; therefore, the ability of dHACM to prevent cardiac damage or promote healing in an in vivo mouse model of acute myocardial infarction was investigated. Acute left ventricular MI was induced in mice by coronary artery ligation. dHACM patches were then sutured to the MI zones, or the infarcted hearts were injected with saline as a control.

After eight weeks post-treatment, dHACM-treated and saline-treated hearts were histologically sectioned and stained with Masson's trichrome to quantify infarct size or with antibodies for c-Kit for recruitment of c-Kit positive stem/progenitor cells, Ki67 for proliferation, TUNEL staining for apoptosis, and CD31 for vascular endothelial cells. Results indicated that dHACM grafts promoted cardiac repair in vivo after MI by reducing the size of fibrotic scarring. An examination of cellular activity demonstrated an increased number of c-Kit positive cells, greater cell proliferation with inhibition of apoptosis, and an increased number of CD31 positive vessels in dHACM-treated cardiac tissue. These results show that treatment with dHACM in this animal model improved cardiac repair following MI through multiple paracrine effects, including through improved blood supply and recruitment of autologous stem cells.

Keywords: dHACM; Amnion; Chorion; Amniotic Membrane; Myocardial Infarction; Stem Cell

Abbreviations: MI: Myocardial Infarction; PPCI: Primary Percutaneous Coronary Intervention; LV: Left Ventricular; LVAD: Left Ventricular Assist Device; dHACM: dehydrated Human Amnion/Chorion Membrane; FDA: Food and Drug Administration; AATB: American Association of Tissue Banks; HIV: Human Immunodeficiency Virus; HTLV: Human T-Lymphotropic Virus; IACUC: Institutional Animal Care and Use Committee; NOD: Non-Obese Diabetic; SCID: Severe Combined Immuno-Deficiency; LAD: Left Anterior Descending; TUNEL: Terminal deoxynucleotidyl transferase dUTP Nick End Labeling; ANOVA: Analysis of Variance; SCF: Stem Cell Factor
\end{abstract}

\section{Introduction}

Acute myocardial infarction (MI), more commonly known as heart attack, is a major consequence of cardiovascular disease and a leading cause of morbidity and mortality worldwide. Acute MI occurs when coronary occlusion causes diminished blood supply to the cardiac tissue, and myocardial ischemia results in irreversible damage or cell death [1]. Reperfusion therapy in the form of primary percutaneous coronary intervention (PPCI) has become the gold standard of treatment for MI and has significantly improved short-term outcomes; however, damage to the cardiac tissue still results in post-MI left ventricular (LV) remodeling. $\mathrm{LV}$ remodeling occurs in response to loss of myocardial function in the form of tissue scarring, dilatation, and hypertrophy, and while remodeling is initially beneficial, this compensatory mechanism can be maladaptive, ultimately leading to heart failure [2]. There are currently about 5.1 million Americans living with heart failure with 825,000 new cases each year. It is estimated that the total direct and indirect costs for heart failure in the United States exceed $\$ 30$ billion each year [3].

Therefore, there is a rising and urgent need for novel therapies for the treatment of MI, particularly to treat or prevent post-MI LV remodeling. Currently, pharmacologics, LV assist 
devices (LVADs), and total heart transplantation are the only forms of treatment, and none of them are without significant limitations. For example, the efficacy of pharmacologics for the prevention and treatment of post-MI LV remodeling and heart failure is limited, LVADs are mainly used as a temporary bridge to cardiac transplantation, and cardiac transplantation faces limited donor availability and is only used as a last course of action. Due to the inability of cardiac muscle to regenerate following injury, stem cell therapy has been widely explored as a treatment for cardiac disease, including numerous ongoing clinical trials; however, significant gains in cardiac function have yet to be established [4-6]. Poor engraftment and low survival rates of injected cells represent major limiting factors in the efficacy of cellular therapies for cardiac repair [4-7].

Dehydrated human amnion/chorion membrane (dHACM) patches (EpiFix ${ }^{\circledR}$ and AmnioFix ${ }^{\circledR}$, MiMedx Group, Marietta, GA) are human allografts comprised of laminated amnion and chorion amniotic membranes derived from the placenta. By using a gentle cleansing and dehydration process (PURION ${ }^{\circledR}$ Process), dHACM retains and preserves the native extracellular matrix architecture and biological activity of the amniotic membrane tissue [8-10]. The properties of PURION Processed dHACM suggest that it may be a promising tissue for use in treatment of acute MI. dHACM contains structural collagen and additional extracellular matrix proteins, as well as a unique array of regenerative cytokines and growth factors, involved in the regulation of wound healing and inflammation [11-14]. These soluble cues remain biologically active in $\mathrm{AHACM}$ and have been shown to promote cell proliferation and endogenous growth factor production, recruit stem and progenitor cells, and promote angiogenesis [11-17]. Amniotic membranes are also known to be immunologically privileged tissues with the ability to reduce pain, prevent scarring, and control inflammation $[18,19]$. Recent clinical trials have established the ability of dHACM to promote healing in a variety of refractory wounds without recurrence in long-term follow-up [20-22], while preliminary results suggest that dHACM may also play a protective role in preventing the progression of osteoarthritis [23]. These properties suggest that dHACM may also possess the ability to either prevent damage or assist cardiac repair following MI.

As a proof-of-concept evaluation, the ability of dHACM to attenuate damage or promote cardiac repair in a mouse model of acute myocardial infarction was investigated, including effects on infarct size and cardiac remodeling. dHACM was examined for its ability to reduce scar formation and inflammation, promote cell proliferation and survival, attract endogenous stem/progenitor cells, and enhance angiogenesis in vivo.

\section{Materials and Methods}

\section{Dehydrated Human Amnion/Chorion Membrane (dHACM)}

dHACM is a dehydrated human allograft comprised of laminated amnion and chorion membranes derived from the placenta [8-10]. Human placentas were donated under informed consent, following Caesarean sections, in compliance with the Food and Drug Administration's (FDA) Good Tissue Practice and American Association of Tissue Banks (AATB) standards. All donors were tested and confirmed to be free of infectious diseases, including human immunodeficiency virus (HIV), human T-lymphotropic virus (HTLV), hepatitis B and C, and syphilis. Amnion and chorion were isolated from placenta and processed with a proprietary PURION® Process that involves gentle cleansing of the layers. The amnion and chorion were then laminated to form the graft, and the graft was dehydrated under controlled drying conditions [10]. Specific versions of dHACM (EpiFix® and AmnioFix $®$, MiMedx Group) were used as the test material in these studies; therefore, the results of these studies apply only to PURION@ Processed dehydrated human amnion/chorion composite grafts (dHACM).

\section{Myocardial Infarct (MI) Model}

A previously established mouse myocardial infarction model was used to examine the efficacy of dHACM in inducing cardiac repair [24-27]. All murine experiments were conducted in accordance with protocols approved by the University of Miami's Institutional Animal Care and Use Committee (IACUC). Either 8-week old female non-obese diabetic (NOD)/severe combined immunodeficiency (SCID) mice or wild type mice, obtained from the Jackson Laboratory (Bar Harbor, ME),were used to examine the effects of human amniotic membrane implants in a mouse xenograft model. Mice were anesthetized, orally intubated, and underwent left thoracotomy in the fourth intercostal space. The left anterior descending branch of the coronary artery (LAD) was ligated using an 8-0 nylon suture to induce MI.

\section{dHACM Treatment}

Following acute myocardial infarction, mice were either treated with a dHACM patch or with saline control treatment. $5 \mathrm{~mm}$ x $5 \mathrm{~mm}$ dHACM patches (EpiFix $\AA$ or AmnioFix $\AA$ ) were sutured to the MI zones. Mice receiving saline injections alone were used as controls. The pericardium was re-draped over the heart, and the chest was closed. Animals were placed into clean cages and housed for the duration of 8weeks after recovery. $\mathrm{n}=10$ mice were used for treatment and control groups, and the investigators were blinded to the type of treatment. Mice that did not recover from the coronary artery ligation due to surgical mortality were excluded from analysis. Animal deaths did not appear to be related to dHACM treatment.

\section{Histological Staining of Infarcts}

Eight weeks post-treatment, mice were euthanized, and analyzed for cardiac remodeling. Hearts from each group were fixed in $10 \%$ formalin, and embedded in paraffin. Paraffin sections were subjected to Masson's Trichrome staining, and quantification of infarct size was performed. The average infarct size was obtained by calculation of the average length of the circumference in the infarct portion and the normal area. These 
indices of cardiac remodeling were compared among dHACM treatment groups and saline controls.

\section{Immunohistochemistry}

To study if dHACM contributes paracrine factors that resulted in enhanced cardiomyocyte proliferation, decreased apoptosis, and induced recruitment of local c-Kit positive cardiac progenitor cells or endogenous stem cells into the infarcted area, tissue sections were stained with antibodies specific for Ki67 (proliferation marker), terminal deoxynucleotidyl transferase dUTP nick end labeling (TUNEL) for apoptosis, and c-Kit, respectively.

To examine vascularization in cardiac tissue, histological tissue sections were also stained with antibodies for CD31 to identify vascular endothelial cells. The sections were counterstained for troponin (red) for myocardial tissue and with DAPI (blue) for cell nuclei. The total number of CD31-positive vessels per $1.0 \times 10^{4} \mu \mathrm{m}^{2}$ of MI area was counted and compared in samples across different groups, as a measure of vascularity.

\section{Statistical Analysis}

Values were reported as mean \pm standard deviation. Statistical analyses were performed using analysis of variance (ANOVA) with Tukey's post hoc test for pair wise comparisons. Significant differences were assigned when $p \leq 0.05$.

Results

\section{dHACM treatment reduced infarct size following MI}

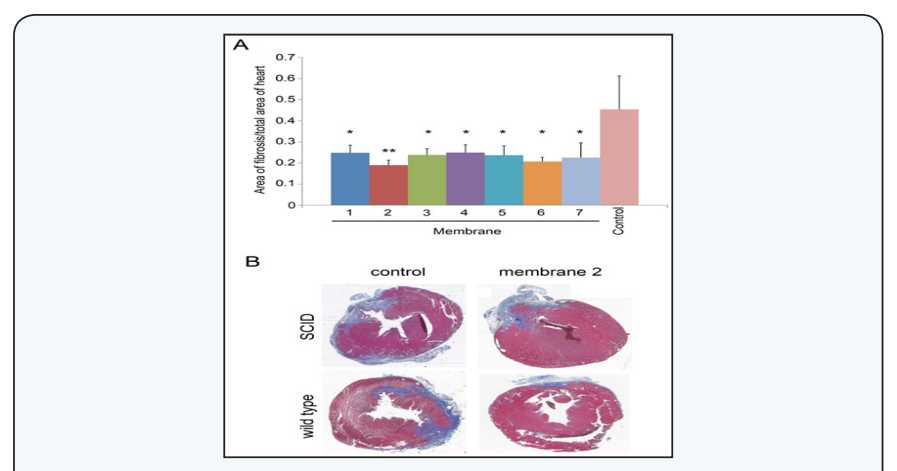

Figure 1: dHACM patches protected mice from damage following experimentally induced myocardial infarction (MI). After 8-weeks following MI, tissue fibrosis was measured through Masson's trichrome staining. Treatment of mice with dHACM significantly reduced infarct size in mice following MI. (A) Quantification of infarct size relative to total area of the heart from membrane treated mice and control treated mice is shown. Membranes 2, 3, 4, and 6 = AmnioFix; Membranes 1, 5, and $7=$ EpiFix. ${ }^{*}$ indicates significantly reduced infarct size, compared to controls $(p \leq 0.05),{ }^{* *}$ indicates significantly reduced infarct size, compared to controls ( $p \leq 0.001)$. (B) Representative Masson's trichrome stained images of dHACM treated (right) and control treated (left) mice. Masson's trichrome stained fibrotic scar tissue blue.

A collagenous scar was visible in the infarct sites, indicating that coronary artery ligation resulted in cardiac fibrosis (Figure 1, blue). dHACM treated mice showed a significant decrease of approximately $50 \%$ in infarct area following MI, compared to control (saline) treated mice ( $\mathrm{p} \leq 0.05$; Figure 1$)$. There was no statistically significant difference in infarct size between the EpiFix and AmnioFix-treated mice; therefore, all dHACM patch results were combined for the following analyses. Additionally, reduction in infarct size was seen in both dHACM-treated NOD/ SCID and wild type mice, indicating that the dHACM tissue was well tolerated by wild type mice.

\section{dHACM treatment recruited c-Kit positive stem cells following MI}

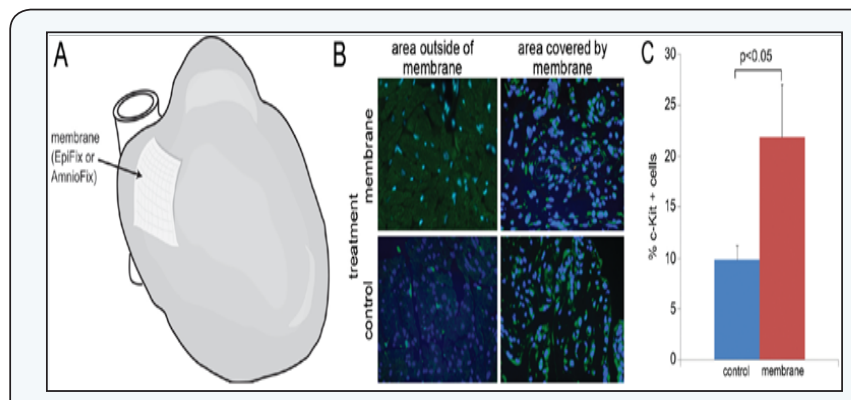

Figure 2: Treatment with dHACM increased the number of c-Kit positive cells, following myocardial infarction. (A) Schematic depiction of the placement of the dHACM patch over the infarcted cardiac tissue. (B) Representative immunostaining for $\mathrm{c}$-Kit in regions of the heart covered by the $\mathrm{dHACM}$ or regions peripheral to the membrane. (C) Quantification of c-Kit positive stem/progenitor cells in the tissue sections demonstrated significantly greater numbers of c-Kit positive cells in dHACM treated tissues, compared to controls, indicative of increased stem cell recruitment $(p \leq 0.05)$.

A significant increase in the stem cell marker c-Kit was observed in dHACM-treated hearts, compared to the control group ( $\mathrm{p} \leq 0.05$; Figure 2 ). Significantly greater numbers of $c$-Kit positive cells were present both in the region directly in contact with the $\mathrm{dHACM}$, as well as regions of the heart peripheral to the membranes. c-Kit is a cell surface receptor for stem cell factor (SCF), and it is a marker for hematopoietic stem cells and cardiac stem/progenitor cells $[28,29]$. Therefore, the increased presence of c-Kit positive cells indicated that $\mathrm{dHACM}$ treatment promoted the recruitment of endogenous c-Kit-positive stem cells either from the bone marrow or locally from the heart to the site of infarction following acute MI.

\section{dHACM treatment promoted cardiomyocyte survival following MI}

There was a significant increase in the cell proliferation marker Ki-67 in the membrane-treated hearts, compared to the control group ( $\mathrm{p} \leq 0.001$; Figure 3$)$. Ki-67 expression also extended beyond the region in direct contact with the membranes into the distal heart tissue. Ki-67 is a nuclear protein that is a marker for proliferating cells [30]. Additionally, there was a significant decrease in apoptosis in the membranetreated hearts, compared to the saline treatment $(\mathrm{p} \leq 0.001)$, as measured by TUNEL staining (Figure 4). TUNEL staining is indicative of DNA fragmentation, correlated to cell apoptosis. 
Increased Ki-67/troponin double-positive cells and decreased TUNEL/troponin double-positive cells indicated that dHACM treatment promoted cardiomyocyte proliferation and inhibited cardiomyocyte apoptosis, concurrently. These data suggest that paracrine signals from the dHACM patches promoted cell proliferation, inhibited apoptosis, and enhanced cell survival following MI.

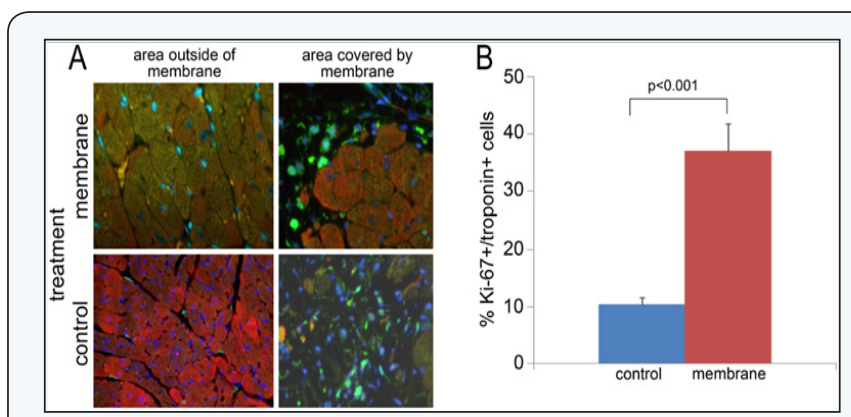

Figure 3: Ki-67 levels were elevated in dHACM-treated heart tissue both in the area covered by the membrane and outside of the membrane-treated area following myocardial infarction. (A) Representative immunostaining of membrane and control-treated hearts. The treated hearts were stained for Ki-67 proliferation marker (green) and cardiac muscle marker troponin (red). Cell nuclei were counterstained with DAPI (blue). (B) Quantification of Ki-67 positive cells demonstrated significantly greater numbers of Ki-67 positive cells in dHACM treated tissues, compared to control treated hearts, indicative of increased proliferation $(p \leq 0.001)$.

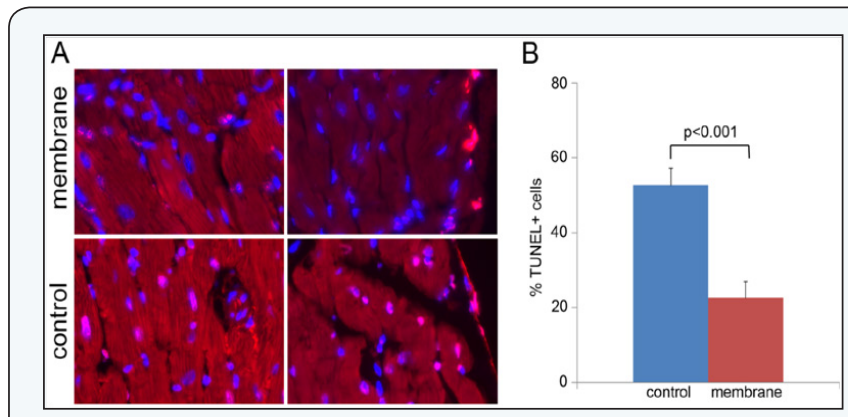

Figure 4: dHACM treatment significantly decreased apoptosis, following myocardial infarction. (A) TUNEL staining (pink) was used to identify apoptotic cells in the membrane and controltreated heart tissue. (B) Quantification of TUNEL positive cells demonstrated significantly fewer TUNEL positive cells in dHACM treated tissues, compared to control hearts, indicative of decreased apoptosis $(p \leq 0.001)$.

\section{dHACM treatment promoted angiogenesis following MI}

An increased number of blood vessels were found in the membrane-treated hearts, compared to the control mice $(\mathrm{p} \leq 0.05)$, as indicated by staining for CD31 positive vessels (Figure 5). CD31 is a cell surface marker for vascular endothelial cells. An increase in CD31 positive vessels indicated enhanced vascularization in the cardiac tissue, suggesting that $\mathrm{dHACM}$ treatment increased angiogenesis and neovascularization within the cardiac tissue following MI.

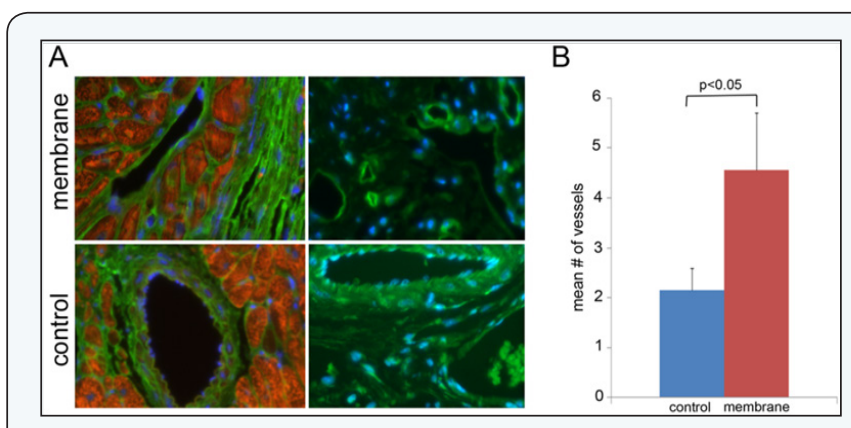

Figure 5: dHACM-treated hearts had significantly increased numbers of blood vessels, compared to control tissues, following myocardial infarction. (A) CD31 staining (green) of the membrane-treated and control hearts indicated a significant increase in the number of CD31 positive blood vessels. The sections were counterstained with troponin (red) and DAPI (blue). (B) Quantification of mean number of vessels per field demonstrated significantly more vessels in dHACM-treated hearts, compared to saline treatment (control), indicative of increased angiogenesis and neovascularization $(p \leq 0.05)$.

\section{Discussion}

Using an established coronary artery ligation model of acute left ventricular myocardial infarction, these results represent proof-of-concept that treatment with dehydrated human amnion/chorion membrane (dHACM) grafts may be capable of promoting cardiac repair in vivo after MI. dHACM treatment reduced the size of fibrotic scarring after MI, indicating that dHACM had a protective effect on the ischemic tissue. Fibrosis of cardiac tissue is an important aspect of cardiac remodeling, as the collagenous scar remains stiff and unable to contract. Cardiac scar tissue leads to further remodeling, including left ventricular hypertrophy, a compensatory mechanism which can ultimately result in heart failure.

As an initial investigation to determine the mechanism of action for dHACM-mediated cardiac repair, an examination of cellular composition and activity in the cardiac tissue was performed to examine a number of events which may promote cardiac repair. An increased number of c-Kit positive cells were identified in dHACM-treated cardiac tissue, suggesting that dHACM may recruit c-Kit positive hematopoietic stem cells and/ or cardiac stem cells to the site of injury, stimulating repair. Additionally, dHACM promoted cell proliferation while inhibiting apoptosis, as demonstrated by Ki-67 and TUNEL staining, respectively, demonstrating enhanced cell survival. Finally dHACM treatment resulted in an increased number of CD31 positive vessels in cardiac tissue after MI, indicative of enhanced angiogenesis. Together these results suggest that treatment with dHACM may improve cardiac repair following MI through multiple paracrine effects. dHACM protected cardiac cells and tissue from damage in vivo, possibly through improved blood supply to the infarcted tissue. Additionally, dHACM recruited autologous stem/progenitor cells into the ischemic cardiac tissue. Recruitment of autologous cardiac stem cells possesses 
tremendous potential to promote repair and regeneration of cardiac tissue.

The properties of dHACM suggest that it may be a potential therapeutic biomaterial for MI treatment as a cardiac patch. dHACM contains a vast array of growth factors and cytokines within a naturally-derived extracellular matrix scaffold. The PURION Process preserves the bioactivity inherent to amniotic membrane tissue, including a unique array of regenerative cytokines and growth factors. To date, over 226 growth factors, cytokines, and regulatory molecules have been identified in dHACM tissues [11-14,31]. These soluble cues remain biologically active in $\mathrm{dHACM}$, combining to promote cell proliferation and endogenous growth factor production of various cell types, recruit stem cells to the site of implantation, and promote angiogenesis in vitro and in vivo [11-17]. dHACM is also inherently biocompatible and non-immunogenic. Amniotic membrane is an immunologically privileged tissue that acts as a natural barrier between the mother and developing fetus, and treatment with amniotic tissue has demonstrated the ability to reduce pain, prevent scarring, and modulate inflammation $[18,19]$. Therefore, dHACM tissue represents a structural patch that contains collagen and extracellular matrix which may also serve as a biologically active bandage for the infarct zone.

This is the first report that $\mathrm{dHACM}$ has positive effects in protecting tissue or promoting repair following acute myocardial infarction. A previous study by Cargnoni et al. [32] demonstrated that fresh human amniotic membrane (used within 24 hours) significantly reduced post-ischemic cardiac injury using a similar rat coronary artery ligation model [32]. In the Cargnoni study, amniotic membrane-treated rats demonstrated preservation of cardiac dimensions and improved cardiac contractile function, in terms of higher left ventricle ejection fraction, fractional shortening, and wall thickening, within 7 days after application and persisting for at least 2 months [32]. Additionally, no engraftment of amniotic cells was detected into host cardiac tissues, suggesting that repair was likely mediated by the release of cardio protective soluble factors to the injured ischemic tissue [32]. Since dHACM used in this study is dehydrated and does not contain viable cells but retains and preserves the bioactive cellular components of the native amniotic membrane, it is likely that the cardiac repair demonstrated here is also mediated through similar soluble signals.

These results suggest that dHACM may warrant further investigation for use in cardiac repair following myocardial infarction. Follow up studies will include demonstrating improvement of important functional outcomes such as left ventricular dimensions, contractility, and ejection volume, as well as demonstrating efficacy in a large animal model of MI, such as a porcine model. Additional experiments are also required to further elucidate the roles of stem cell recruitment and neovascularization in cardiac repair, including potential interactions and characterization of the molecular mechanisms underlying the efficacy of dHACM in the repair of other tissues.
However, this work is a first step suggesting that dHACM allografts possess tremendous potential in promoting cardiac repair following acute myocardial infarction.

\section{Conclusion}

dHACM allografts appeared to promote cardiac repair after MI by reducing the size of fibrotic scarring in a mouse model. Application of dHACM patches stimulated an increase in c-Kit positive stem cells, cell survival, and vascularization in infarcted cardiac tissue. These results suggest that treatment with dHACM following MI may improve cardiac repair through multiple paracrine effects, including through improved blood supply and recruitment of autologous stem cells.

\section{Acknowledgement}

This work was performed at University of Miami Miller School of Medicine by Chunming Dong, MD, FACC and Shoukang Zhu, MD. The authors thank Anna Fallon, PhD for critical comments on an earlier version of the manuscript.

\section{Conflict of Interest}

MiMedx funded the study with the University of Miami. JJL and TJK are employees of MiMedx. JF is a consultant for MiMedx.

\section{References}

1. Boersma E, Mercado N, Poldermans D, Gardien M, Vos J, et al. (2003) Acute myocardial infarction. Lancet 361(9360): 847-858.

2. Sutton MGSJ, Sharpe N (2000) Left ventricular remodeling after myocardial infarction: pathophysiology and therapy. Circulation 101(25): 2981-2988.

3. Go AS, Mozaffarian D, Roger VL, Benjamin EJ, Berry JD, et al. (2014) Heart disease and stroke statistics--2014 update: a report from the American Heart Association. Circulation 129(3): e28-e292.

4. Wu KH, Mo XM, Han ZC, Zhou B (2011) Stem cell engraftment and survival in the ischemic heart. Ann Thorac Surg 92(5): 1917-1925.

5. Pittenger MF, Martin BJ (2004) Mesenchymal stem cells and their potential as cardiac therapeutics. Circ Res 95(1): 9-20.

6. Muller Ehmsen J, Krausgrill B, Burst V, Schenk K, Neisen UC, et al. (2006) Effective engraftment but poor mid-term persistence of mononuclear and mesenchymal bone marrow cells in acute and chronic rat myocardial infarction. J Mol Cell Cardiol 41(5): 876-884.

7. Le Blanc K and Pittenger M (2005) Mesenchymal stem cells: progress toward promise. Cytotherapy 7(1): 36-45.

8. Daniel J, Tofe R, Spencer R, et al., inventors (2013) MiMedx Group, Inc (Kennesaw, GA), assignee. Placental tissue grafts. U.S. Patent 8,357,403.

9. Daniel J, inventor (2013) MiMedx Group, Inc (Kennesaw, GA), assignee. Placental tissue grafts. U.S. Patent 8,372,437.

10. Daniel J, Tofe R, Spencer R, et al., inventors (2012) MiMedx Group, Inc (Kennesaw, GA), assignee. Placental tissue grafts. U.S. Patent 8,409,626.

11. Koob TJ, Rennert R, Zabek N, Massee M, Lim JJ, et al. (2013) Biological properties of dehydrated human amnion/chorion composite graft: implications for chronic wound healing. Int Wound J 10(5): 493-500.

12. Koob TJ, Lim JJ, Massee M, Zabek N, Denozière G (2014) Properties of dehydrated human amnion/chorion composite grafts: Implications for wound repair and soft tissue regeneration. J Biomed Mater Res B Appl Biomater 102(6): 1353-1362. 
13. Koob TJ, Lim JJ, Massee M, Zabek N, Rennert R, et al. (2014) Angiogenic properties of dehydrated human amnion/chorion allografts: therapeutic potential for soft tissue repair and regeneration. Vasc Cell 6: 10 .

14. Lei J, Priddy LB, Lim JJ, Massee M, Koob TJ (2016) Identification of extracellular matrix components and biological factors in micronized dehydrated human amnion/chorion membrane. Adv Wound Care (New Rochelle). DOI: 10.1089/wound.2016.0699.

15. Maan ZN, Rennert RC, Koob TJ, Januszyk M, Li WW, et al. (2015) Cell recruitment by amnion chorion grafts promotes neovascularization. J Surg Res 193(2): 953-962.

16. Massee M, Chinn K, Lei J, Lim JJ, Young CS, et al. (2016) Dehydrated human amnion/chorion membrane regulates stem cell activity in vitro. J Biomed Mater Res B Appl Biomater 104(7): 1495-1503.

17. Massee M, Chinn K, Lim JJ, Godwin L, Young CS, et al. (2016) Type I and II Diabetic Adipose-Derived Stem Cells Respond In Vitro to Dehydrated Human Amnion/Chorion Membrane Allograft Treatment by Increasing Proliferation, Migration, and Altering Cytokine Secretion. Adv Wound Care (New Rochelle) 5(2): 43-54.

18. Ramakrishnan KM, Jayaraman V (1997) Management of partialthickness burn wounds by amniotic membrane: a cost-effective treatment in developing countries. Burns 23 Suppl 1: S33-S36.

19. Niknejad H, Peirovi H, Jorjani M, Ahmadiani A, Ghanavi J, et al. (2008) Properties of the amniotic membrane for potential use in tissue engineering. Eur Cell Mater 15: 88-99.

20. Zelen CM, Serena TE, Denoziere G, Fetterolf DE (2013) A prospective randomised comparative parallel study of amniotic membrane wound graft in the management of diabetic foot ulcers. Int Wound J 10(5): 502-507.

21. Forbes J and Fetterolf DE (2012) Dehydrated amniotic membrane allografts for the treatment of chronic wounds: a case series. J Wound Care 21(6): 290, 292, 294-296.

22. Sheikh ES, Sheikh ES, Fetterolf DE (2014) Use of dehydrated human amniotic membrane allografts to promote healing in patients with refractory non healing wounds. Int Wound J 11(6): 711-717.
23. Willett NJ, Thote T, Lin AS, Moran S, Raji Y, et al. (2014) Intra-articular injection of micronized dehydrated human amnion/chorion membrane attenuates osteoarthritis development. Arthritis Res Ther 16(1): R47.

24. Graham RM, Thompson JW, Wei J, Bishopric NH, Webster KA (2007) Regulation of Bnip3 death pathways by calcium, phosphorylation, and hypoxia-reoxygenation. Antioxid Redox Signal 9(9): 1309-1315.

25. Wei J, Wang W, Chopra I, Li HF, Dougherty CJ, et al. (2011) c-Jun $\mathrm{N}$-terminal kinase (JNK-1) confers protection against brief but not extended ischemia during acute myocardial infarction. J Biol Chem 286(16): 13995-14006.

26. Wu D, Mennerich D, Arndt K, Sugiyama K, Ozaki N, et al. (2009) Comparison of microsomal prostaglandin E synthase-1 deletion and COX-2 inhibition in acute cardiac ischemia in mice. Prostaglandins Other Lipid Mediat 90(1-2): 21-25.

27. Wu D, Mennerich D, Arndt K, Sugiyama K, Ozaki N, et al. (2009) The effects of microsomal prostaglandin E synthase-1 deletion in acute cardiac ischemia in mice. Prostaglandins Leukot Essent Fatty Acids 81(1): 31-33.

28. Edling CE, Hallberg B (2007) c-Kit--a hematopoietic cell essential receptor tyrosine kinase. Int J Biochem Cell Biol 39(11): 1995-1998.

29. Magenta A, Avitabile D, Pompilio G, Capogrossi MC (2013) c-kitPositive cardiac progenitor cells: the heart of stemness. Circ Res 112(9): 1202-1204.

30. Scholzen T, Gerdes J (2000) The Ki-67 protein: from the known and the unknown. J Cell Physiol 182(3): 311-322.

31. Lim JJ and Koob TJ (2016) Placental Cells and Tissues: The Transformative Rise in Advanced Wound Care. In: C. Fonseca (Editor), Worldwide Wound Healing - Innovation in Natural and Conventional Methods. InTech, Rijeka, Croatia, pp. 121-151.

32. Cargnoni A, Di Marcello M, Campagnol M, Nassuato C, Albertini A, et al. (2009) Amniotic membrane patching promotes ischemic rat heart repair. Cell Transplant 18(10): 1147-1159.

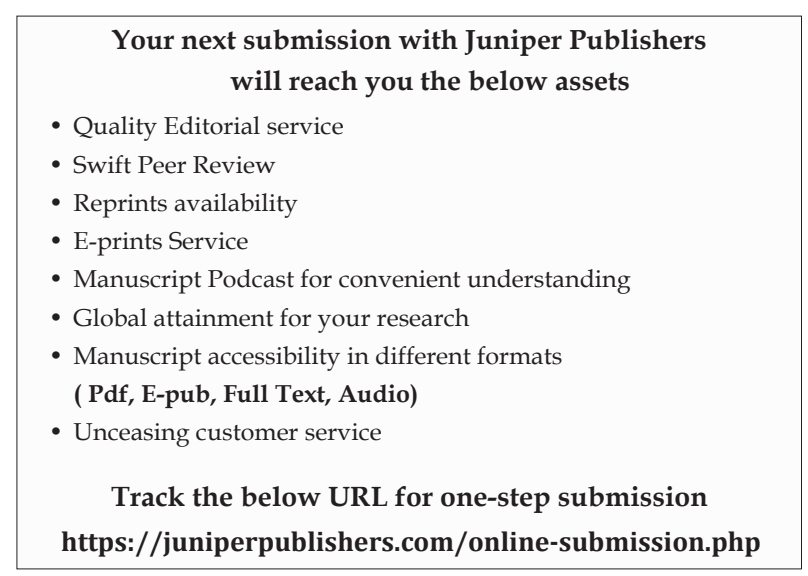

